\title{
El Realismo Científico y la Inducción Pesimista: un debate abierto
}

Damián Islas Mondragón

Universidad Juárez del Estado

de Durango, México 


\title{
El Realismo Científico y la Inducción Pesimista: un debate abierto*
}

\begin{abstract}
Resumen: el debate entre los defensores de ciertas versiones del Realismo Científico y los autores que argumentan a favor de la inducción pesimista, sigue abierto. Este estudio fue hecho con el objetivo de mostrar que el argumento de la inducción pesimista representa un reto serio para estas versiones del Realismo Científico. En la primera parte del texto revisaré algunas de las diferentes maneras de caracterizar la inducción pesimista, enfatizando el tipo de compromiso realista con el que discuten, esto es, si es de índole semántico, epistémico o metafísico. En la segunda parte analizo tres estrategias que se han desarrollado para socavar la inducción pesimista, así como los contra argumentos formulados por los defensores de la inducción pesimista ante estos ataques. Muestro que ninguna de estas tres estrategias logra su objetivo. Termino sugiriendo una posible línea de investigación sobre el tema.
\end{abstract}

Palabras clave: Inducción Pesimista, Realismo Científico, Éxito empírico, Alternativas no concebidas.

\section{Scientific Realism and the Pessimistic Induction: an open debate}

\begin{abstract}
Scientific Realism and the authors who argue in favor of the pessimistic induction, remains open. This study was made with the objective of showing that the pessimistic induction represents a serious challenge for these versions of Scientific Realism. In the first part of the text I will review some of the different ways to characterize the pessimistic induction, emphasizing the type of realistic commitment with which they argue, that is, whether it is of a semantic, epistemic or metaphysical nature. In the second part I analyze three strategies that have been developed to undermine the pessimistic induction, as well as the counter arguments formulated by the defenders of the pessimistic induction before these attacks. I show that none of these three strategies achieves its objective. I conclude by suggesting a possible line of research on the subject.
\end{abstract}

Keywords: Pessimistic Induction, Scientific Realism, Empirical Success, Unconceived Alternatives.

Fecha de recepción: 22 de febrero de 2019

Fecha de aceptación: 20 de marzo de 2019

Forma de citar (APA): Islas-Mondragón, D. (2019). El Realismo Científico y la Inducción Pesimista: un debate abierto. Revista Filosofía UIS, 18(2), doi: 10.18273/revfil.v18n22019004

Forma de citar (Harvard): Islas-Mondragón, D. (2019). El Realismo Científico y la Inducción Pesimista: un debate abierto. Revista Filosofía UIS, 18(2), 53-69.

Damián Islas Mondragón: mexicano. Postdoctorado en Filosofía. Docente e Investigador del Instituto de Ciencias Sociales. Universidad Juárez del Estado de Durango, México.

Correo electrónico: damianislas@ujed.mx

ORCID: 0000-0001-8538-6835

*Artículo de reflexión derivado de investigación. 


\section{El Realismo Científico y la Inducción Pesimista: un debate abierto}

\section{Introducción}

El Realismo Científico (de aquí en adelante RC) es una postura filosófica heterogénea sobre la ciencia defendida no solo por filósofos; sino también por científicos que reflexionan sobre su propio quehacer. De acuerdo con RC, algunas teorías científicas exhiben ciertos rasgos semánticos, epistémicos o metafísicos que se preservan durante el cambio teórico. La inducción pesimista (de aquí en adelante IP), es un argumento que se ha formulado como un reto para algunas versiones de RC. De acuerdo con IP, cualesquiera que sean las virtudes semánticas, epistémicas o metafísicas de las teorías científicas, nada nos garantiza, desde un punto de vista inductivo, que tales rasgos se preserven durante el cambio diacrónico de la ciencia. Por ejemplo, para algunas versiones de RC, el éxito empírico que exhiben las mejores teorías científicas es un rasgo a favor de esta postura. Sin embargo, IP ha argumentado a este respecto que la mayoría de las teorías científicas que en el pasado se consideraron exitosas a un nivel empírico, actualmente han sido abandonadas. De manera que nada nos impide realizar el salto inductivo e inferir que, probablemente, algunas de las teorías que actualmente exhiben éxito empírico, también serán abandonadas en un futuro.

Para solventar este y otros retos que IP le plantea a RC, se han propuesto varias estrategias. De entre estas, destacan tres, a saber, i) minimizar el número y relevancia de las teorías científicas pasadas que fueron exitosas y que en la actualidad se han considerado se han abandonado, ii) distinguir las partes responsables del éxito de las teorías pasadas de las partes ajenas a este éxito y, iii) mostrar que IP es un argumento falaz.

En la primera parte del texto, con el objetivo de contextualizar el problema, revisaré algunas de las diferentes maneras de caracterizar IP, enfatizando el tipo de compromiso realista con el que discuten, esto es, si es de índole semántico, epistémico o metafísico. En la segunda parte analizo las tres estrategias antes mencionadas que se han desarrollado para rechazar algunas versiones de la inducción pesimista. A manera de diálogo, presento algunos contra argumentos 
formulados por los defensores de IP para sostener su postura. A lo largo del texto, muestro que ninguna de estas tres estrategias logra socavar IP de manera contundente. Termino sugiriendo una posible línea de investigación sobre el tema.

\section{Diversas concepciones del argumento de la inducción pesimista}

Es bastante conocida la frase que ideó Putnam para caracterizar la fuerza que supuestamente tiene RC. Me refiero al argumento del (no) milagro: "El Realismo Científico es la única filosofía que no convierte el éxito de la ciencia en un milagro" (Putnam, 1975, p. 73). En realidad, la frase de Putnam solo refleja una de las varias tesis que puede ser defendida por los realistas. Aquí mencionaremos otras dos tesis igualmente fundamentales para el Realismo Científico, a saber:

a. La tesis metafísica: el mundo tiene una estructura definitiva que es independiente de la mente humana.

b. La tesis semántica: las teorías científicas son descripciones condicionadas por la verdad de sus dominios de investigación, por lo que pueden ser verdaderas o falsas.

c. La tesis epistémica (a la que se refiere Putnam): las teorías científicas exitosas a un nivel predictivo están bien confirmadas. Las entidades postuladas por estas teorías, existen en el mundo natural.

Aquí no discutiré cuál de estas tesis es la que mejor caracteriza a RC. Lo que deseo señalar es que las distintas versiones de IP, que a continuación revisaremos, atacan una $u$ otra tesis defendida por RC.

Una formulación temprana de IP puede encontrarse en los trabajos del físico francés Henry Poincaré quien argumentaba en torno a la "ruina de la ciencia", cito a Poincaré:

La naturaleza efímera de las teorías científicas nos toma por sorpresa. Su breve periodo de prosperidad termina, una tras otra vemos su abandono; vemos ruinas apiladas sobre ruinas; predecimos que las actuales teorías, a su vez, sucumbirán en poco tiempo y, concluimos, que fueron creadas absolutamente en vano (1905, p. 160) ${ }^{2}$.

Larry Laudan y Hilary Putnam retomaron el tema a mediados de la segunda mitad del siglo pasado. Cito a ambos autores respectivamente:

\footnotetext{
${ }^{1}$ Para un análisis de las implicaciones de algunas de estas tesis y de los distintos tipos de realismos científicos, véase Psillos (1999).

${ }^{2}$ Todas las traducciones del inglés al castellano son propias.
} 
La mayoría de las teorías científicas del pasado son sospechosas de ser falsas; presumiblemente tenemos razones para anticipar que las teorías de la ciencia actuales sufrirán la misma suerte (Laudan, 1977, p. 126).

¿Qué pasaría si todas las entidades teóricas postuladas por una generación de teorías científicas (moléculas, genes, electrones) no existieran desde el punto de vista de la ciencia futura? ¿Cómo saber que no estamos en un error ahora? Esta es una pregunta sería porque da lugar a la siguiente metainducción: así como ningún término utilizado en la ciencia de hace 50 años, actualmente refiere; ningún término utilizado en la ciencia actual refiere (Putnam, 1978, pp. 24-25).

En la actualidad, la discusión acerca de la validez e importancia de IP, como un argumento serio en contra de ciertas versiones de RC, está siendo ampliamente discutido en la filosofía de la ciencia. Por ejemplo, Seungbae Park se enfoca, como lo hizo Laudan, en el aspecto semántico de las teorías científicas pasadas, a saber, su falsedad: "Dado que las teorías científicas pasadas resultaron ser falsas, lo mismo les sucederá a las teorías científicas actuales" (2014, p. 5).

Michael Devitt discute, como lo hizo Putnam, la falta de referencia de ciertas entidades que postulan algunas teorías científicas, en particular, las entidades inobservables. Sin embargo, al tema metafísico de la referencia, Devitt le agrega otro tema de índole semántico de no menos importancia, a saber, el de la verdad:

Los inobservables postulados por las teorías del pasado no existen; entonces, probablemente los inobservables postulados por las teorías actuales no existen [...] Las teorías científicas del pasado no son aproximadamente verdaderas; entonces, probablemente las teorías actuales no sean aproximadamente verdaderas (2007, p. 14).

Por su parte, Brad Wray ha atacado un rasgo epistemológico defendido por los realistas científicos, a saber, el éxito de las teorías científicas:

Muchas teorías que alguna vez tuvieron éxito han sido descartadas sobre la base de que son falsas. Esta reflexión sobre la historia de la ciencia parece proporcionar una convincente evidencia inductiva de que las teorías exitosas que hoy aceptamos, probablemente sean descartadas en el futuro. Tanto los realistas como los anti-realistas consideran esto como uno de los argumentos más convincentes en apoyo del anti-realismo (2013, p. 4321).

Greg Frost-Arnold enfatiza la aparente conexión entre el éxito empírico y la verdad de las teorías científicas: 
Todas (o "la mayoría") de nuestras teorías científicas pasadas (fundamentales) no son aproximadamente verdaderas, incluso las que fueron empíricamente exitosas en su tiempo. Por lo tanto, todas (o "la mayoría") de nuestras teorías científicas actuales (fundamentales) probablemente no sean aproximadamente verdaderas, incluso las que ahora son empíricamente exitosas (2011, p. 1131).

A este respecto, Laudan no solo rechaza la conexión entre el éxito empírico y la verdad de las teorías científicas. También rechaza cualquier conexión de las teorías científicas y el éxito referencial de las entidades observables e inobservables que postulan, y argumenta cinco razones: a) existen casos de teorías científicas que se supusieron aproximadamente verdaderas y que no fueron empíricamente exitosas; b) existen casos de teorías empíricamente exitosas que no eran aproximadamente verdaderas; c) una teoría que genuinamente refiere puede no ser exitosa a un nivel empírico; d) existen numerosas teorías que fueron empíricamente exitosas que no referían genuinamente y e) existieron teorías que hoy se sabe que son falsas y que tuvieron un alto grado de éxito en diferentes aplicaciones.

A mediados de la década pasada, Kyle Stanford propuso una perspectiva diferente de abordar el tema. De acuerdo con este autor, aunque algunas teorías científicas puedan considerarse suficientemente confirmadas, todavía queda por resolverse el tema de las alternativas no concebidas:

Recordemos que la inducción pesimista clásica nos hace notar simplemente que las teorías exitosas del pasado resultaron ser falsas y sugiere que no tenemos razón para pensar que las actuales teorías exitosas no sufrirán la misma suerte. Por el contrario, propongo que lo que llamo la nueva inducción pesimista de la historia de la ciencia [...] podría concebir sólo una o dos teorías que estuvieron bien confirmadas por la evidencia disponible, mientras que la investigación futura nos revelaría de manera rutinaria [si no es que invariablemente] algunas alternativas radicalmente distintas que también estarían bien confirmadas por la evidencia previamente disponible [...] (Stanford, 2006, p. 19, énfasis en el original).

Por lo tanto, de acuerdo con Stanford: "La historia de la investigación científica ofrece un fundamento racional para pensar que, por lo general, existen alternativas a nuestras mejores teorías científicas que están igualmente bien confirmadas por la evidencia, incluso cuando no podemos concebirlas en este momento" (2006, p. 20).

El argumento de Stanford implica que a pesar de que algunas teorías actuales están epistémicamente bien confirmadas, esto no significa que sean verdaderas (2006, p. 8) debido a que una teoría actualmente no concebida, puede ser empíricamente equivalente, aunque radicalmente distinta, a estas. En otras palabras, no importa cuán impresionantes sean los logros predictivos que exhiba 
una teoría científica, tales logros no favorecen la teoría en cuestión por encima de otras teorías distintas que también muestren los mismos logros predictivos. De ser este el caso, un nuevo problema surge para la postura realista, esto es, establecer los criterios adecuados para determinar, llegado el momento, cuál de las teorías empíricamente equivalentes debe ser abandonada y cuál debe conservarse durante el cambio teórico ${ }^{3}$.

Finalmente, mencionaré otra manera reciente de concebir IP. De acuerdo con Peter Vickers, a la conocida noción de predicción empírica debemos agregarle un elemento relativamente novedoso, a saber, los working posits: "Existen varios ejemplos de teorías en la historia de la ciencia que lograron un éxito predictivo muy significativo, pero en las cuales ciertas partes esenciales no son aproximadamente verdaderas" (2017, p. 3223).

Considerando estas diferentes maneras de concebir IP, las cuales —por supuesto- no son exhaustivas ${ }^{4}$, en las siguiente sección analizaré tres estrategias que se han desarrollado para rechazar ciertas versiones de IP. Conforme presente cada una de estas estrategias, también analizaremos algunos de los contra argumentos que los defensores de IP han elaborado para sostener su postura.

\section{Tres estrategias realistas en contra de IP}

Para solventar algunos de los retos que IP le plantea a RC, se han propuesto varias estrategias. Entre estas, destacan tres: minimizar el número y relevancia de las teorías científicas pasadas que fueron exitosas y que actualmente han sido abandonadas. Distinguir las partes responsables del éxito de las teorías pasadas de las partes ajenas a este éxito y mostrar que durante el cambio teórico solo las últimas son desechadas. Mostrar que IP es un argumento falaz. Analicemos los argumentos implícitos en cada una de estas estrategias:

\section{a. Minimizando el número y relevancia de las teorías científicas pasadas que fueron exitosas y que actualmente han sido abandonadas}

Esta estrategia tiene como eje central la conocida lista de teorías científicas que, de acuerdo con Laudan, una vez fueron exitosas empíricamente; no obstante haber contenido términos que no tuvieron referencia en el mundo natural. Algunas de estas teorías son las siguientes:

\footnotetext{
${ }^{3}$ A este argumento también se le ha denominado el argumento de la "subdeterminación" de las teorías por la evidencia.

${ }^{4}$ Agradezco el agudo comentario de un revisor anónimo quien precisó que no todos los autores que han defendido IP lo han hecho intentando atacar alguna postura realista. Ciertamente, como argumenta Seungbae Park, IP puede dirigirse en contra de i) teorías científicas, ii) científicos, iii) realistas e, incluso, iv) teorías anti-realistas; como intenta hacerlo el mismo Park (2017).
} 
-Las esferas cristalinas de la astronomía antigua y medieval

- La teoría humoral de la medicina

- La teoría del efluvio de la electricidad estática

- La geología catastrófica

- La teoría química del flogisto

-La teoría calórica

- La teoría vibratoria del calor

- La teoría de la fuerza vital de la fisiología

-El éter electromagnético

-El éter óptico

- La teoría de la inercia circular

-La teoría de la generación espontánea (Laudan, 1981, p. 33)5.

Una manera de minimizar el número y relevancia de las teorías que integran el listado elaborado por Laudan es descalificar su pertinencia. Este argumento puede ser formulado de la siguiente manera:

Argumento realista a. 1 : puede ponerse en duda que la mayoría de las teorías citadas por Laudan hayan sido teorías genuinamente exitosas debido a que no lograron hacer predicciones novedosas. Una de las razones de lo anterior es que no formaron parte de la ciencia "madura" o suficientemente "refinada" de su tiempo (Psillos, 1999, pp. 99-100 y McMullin, 1984, pp. 17-18).

Contrargumento IP al argumento realista a.1: ante este argumento realista, algunos defensores de IP como Larry Laudan han sostenido que, si la noción de 'éxito' científico se liga a la noción de 'predicción empírica novedosa', entonces no solo las teorías científicas "maduras" han hecho predicciones novedosas. Ciertamente, a pesar de que la teoría del flogisto no exhibió referencias genuinas, podemos considerar que con esta teoría se hicieron predicciones exitosas, por ejemplo, con relación a las propiedades reductoras de lo que Henry Cavendish llamó "aire o gas inflamable" (lo que hoy conocemos como hidrógeno). Si este argumento es correcto, los realistas tendrían que aceptar que los teóricos del flogisto a la vez que defendieron ideas francamente erróneas, simultáneamente hicieron predicciones correctas; lo que convertiría el éxito predictivo de ciertas teorías científicas "inmaduras", en un misterio. Por lo anterior, IP puede argumentar que la distinción entre las ciencias "maduras" y las "inmaduras" a la que apelan los realistas, no es determinante para el éxito predictivo que exhibe la ciencia.

Otra manera de minimizar el número y relevancia de las teorías que integran el listado elaborado por Laudan es mostrar su parcialidad. Este argumento puede ser formulado de la siguiente manera:

${ }^{5}$ Esta lista la siguen suscribiendo autores como Carsten Held (2011). 
Argumento realista a.2: la lista de teorías construida por Laudan es parcial por ser deficientemente aleatoria y poco representativa. Este argumento lo suscriben autores como Mizrahi (2013); Fahrbach (2011) y de Solla (1963).

De acuerdo con Mizrahi, la lista de Laudan solo muestra teorías exitosas del pasado distante y no del pasado reciente, veamos por qué: de 124 ejemplos de teorías obtenidas del Oxford Reference Online sobre tópicos de Biología, Química, Física e Historia de la Ciencia Moderna, Mizrahi seleccionó aleatoriamente 40 teorías. De esta muestra, solo el $27 \%$ son consideradas actualmente teorías abandonadas ${ }^{6}$. Por lo tanto, este rastreo muestra el escaso fundamento empírico de IP. Si este argumento es correcto, sostiene Mizrahi, en realidad tendríamos mejores razones para defender una inducción optimista $(\mathrm{IO})$ que una inducción pesimista (Mizrahi, 2013, p. 3223).

Por su parte, de Solla y Fahrbach han argumentado, respectivamente, que los defensores de IP tampoco toman en cuenta el crecimiento exponencial de la ciencia. A este respecto, de Solla afirma que alrededor del $85 \%$ de todos los científicos que han existido, lo están haciendo ahora. Pero no solo el número de científicos se ha incrementado, también el número de artículos científicos producidos en los últimos 300 años se ha duplicado cada 15-20 años. Si esto es así, entonces aproximadamente el 50\% de la ciencia producida durante este período, se ha hecho en los últimos 15-20 años y alrededor del 96\% se ha hecho en los últimos 80 años. Por lo anterior, sostiene de Solla, la ciencia que podemos llamar 'contemporánea' representa un porcentaje mucho más alto de la que podemos llamar 'historia de la ciencia' (de Solla, 1963, p. 1).

Siguiendo esta línea argumentativa, Fahrbach sostiene que alrededor del 95\% de la ciencia realizada en este período de 300 años, se realizó después del año 1915 y alrededor del 80\% se ha realizado después del año 1950 (Fahrbach, 2011, p. 148). Si estas cifras son correctas, un cálculo rápido nos indica que si el $85 \%$ de las teorías de los últimos cuarenta años se hubieran abandonado7, todavía la mayoría de las teorías que se han desarrollado son actualmente aceptadas dado que este $85 \%$ representa solo el $21 \%$ del total de todas las teorías científicas desarrolladas durante este período. De ser correcto este argumento, al parecer tenemos más razones para IO que para IP. Y dado que IP es un argumento cuya base inductiva recae en la historia de la ciencia; no podemos inferir la suerte que correrá la mayoría de las teorías científicas actualmente aceptadas a partir de una base tan poco representativa del "total" de la ciencia moderna.

\footnotetext{
${ }^{6}$ Se contemplan las teorías abandonadas bajo el supuesto de que la razón de su abandono pudo haber sido su falsedad.
}

${ }^{7}$ Tomemos en cuenta que el listado sugerido por Laudan fue elaborado en 1981. 
Contrargumento IP al argumento realista a.2: este contraargumento a las tesis desarrolladas por Mizrahi, de Solla y Fahrbach se lo debemos a Brad Wray. En realidad, es un argumento muy simple. De acuerdo con este autor, los científicos que trabajaron, por ejemplo, entre 1878 y 1898 pudieron considerarse responsables del $50 \%$ de la ciencia producida hasta ese momento (según las mismas cifras presentadas por de Solla y Fahrbach). Incluso, pudieron considerar que solo el $27 \%$ de las teorías desarrolladas hasta ese momento habían sido desechadas (como argumenta Mizrahi). Lo mismo puede decirse de los científicos que trabajaron, por ejemplo, entre 1838 y 1858. Pero ahora sabemos que casi todas las teorías desarrolladas entre 1838 y 1898 fueron abandonadas (quizá por ser falsas). Por lo tanto, los científicos de cualquier época pudieron formular el mismo argumento que en la actualidad formulan de Solla, Fahrbach y Mizrahi sin que ello signifique que el crecimiento exponencial de la ciencia justifique $I O$ (Wray, 2013, p. 4326).

Notemos que el argumento de Wray deja abierta la posibilidad de que las teorías pertenecientes a cualquier periodo de la ciencia hayan sido, de hecho, exitosas a un nivel empírico; como argumenta Laudan. De manera que, para bloquear IP, lo que los realistas requieren es identificar uno o más rasgos específicos que las actuales teorías posean y que las anteriores teorías no tengan. Encontrar este rasgo es el objetivo de la segunda estrategia realista en contra de IP que examinaremos a continuación.

\section{b. Distinguir las partes responsables del éxito de las teorías pasadas de las partes ajenas a este éxito y mostrar que durante el cambio teórico solo las últimas son desechadas}

Una manera de señalar las partes responsables del éxito empírico de las teorías es identificando qué elementos de las teorías pasadas se han conservado durante el cambio diacrónico de la ciencia. Este argumento puede ser formulado de la siguiente manera: argumento realista b.1: no todos los constituyentes de las teorías científicas contribuyen de la misma manera a su éxito empírico. Este argumento lo suscriben autores como Bird (2007); Psillos (1999); Kitcher (1995) y Leplin (1992).

Ciertamente, no todos los constituyentes teóricos contribuyen —o contribuyen de la misma manera- con el éxito empírico de una teoría científica ((Psillos, 1999, p. 108). Existen ciertos rasgos de las teorías científicas que son centrales para lograr su éxito empírico, mientras que otros son secundarios o periféricos a este éxito. En este sentido, se ha sugerido que algunos de estos rasgos centrales son los controles metodológicos más estrictos que hoy se poseen, especialmente en relación a la capacidad de las teorías científicas de hacer predicciones novedosas (Kitcher, 1995, p. 614). Y se afirma que, una vez que se han identificado los rasgos que hacen que una teoría científica sea exitosa, se preferirán teorías que 
presenten estos rasgos a teorías que no lo hagan (Leplin, 1992, pp. 440-442). En este sentido, Alexander Bird ha afirmado que la IP de Laudan inferida de su lista es dudosa porque las teorías científicas no son inventadas independientemente de los éxitos y errores cometidos por las teorías predecesoras (Bird, 2007, p. 80). Las actuales teorías científicas poseen, se argumenta, instrumentos metodológicos que quizá fueron impensables para las generaciones anteriores, con los cuales se ha logrado un grado de precisión en los procedimientos de medición y predicción científica nunca antes alcanzados.

Contrargumento IP al argumento realista b.1: una teoría científica puede exhibir diferentes éxitos explicativos. Veamos un ejemplo. La teoría calórica, al parecer, explicó exitosamente los siguientes fenómenos:

-La tendencia del calor hacia el equilibrio.

-La expansión de la materia por el calor.

-El calor latente en los cambios de estado de la materia.

-La elasticidad de los gases y la fluidez de los líquidos.

-El calor que se pierde y que se absorbe en algunas reacciones químicas.

-La combustión.

-La radiación del calor (Chang 2003, p. 907).

Si esto es así, esta pluralidad de éxitos empíricos, incluso en una teoría científica que hoy se considera falsa como la calórica, hace difícil distinguir entre los constituyentes responsables de los no responsables de sus éxitos empíricos. Cuando una teoría científica solo exhibe uno o dos éxitos empíricos, la tarea de ubicar los constituyentes "responsables" de tales éxitos parece ser manejable. Sin embargo, cuando una teoría como la calórica exhibe tal cantidad de éxitos empíricos, la misma tarea parece complicarse.

Por otro lado, los defensores de IP también pueden argumentar que en pocas ocasiones una hipótesis aislada nos conduce a predicciones empíricas exitosas. Casi siempre las hipótesis científicas centrales están acompañadas de hipótesis y supuestos auxiliares, ciertas condiciones iniciales y teorías relacionadas ${ }^{8}$. Si esto es así, no es fácil "responsabilizar" del éxito —o del fracaso— de una teoría científica a uno solo de los elementos que las constituyen. Cada uno de estos aspectos estaría tan estrechamente relacionado con los otros, que parece imposible aislar a uno solo para explicar el éxito empírico de la teoría. Finalmente, desde un punto de vista cualitativo, tratar de determinar y evaluar el grado con el que cada uno de estos aspectos "contribuye" al éxito de la teoría también parece ser una tarea complicada.

${ }^{8}$ Este argumento es conocido como la tesis Duhem-Quine. 
Asimismo, Michael Friedman ha argumentado que no está claro a que se refieren los realistas cuando argumentan que las teorías actuales "preservan" algunos constituyentes de las teorías pasadas responsables de sus éxitos empíricos. Si se refieren a algunas técnicas de razonamiento, incluyendo algunas estructuras matemáticas como ha sugerido John Worral (1989, p. 120), tales aspectos parecen ser relativos a los marcos teórico-conceptuales aceptados en un momento determinado. En efecto, la historia de la ciencia nos indica que no existe ningún marco a priori que establezca de una vez y para siempre las condiciones necesarias para que el conocimiento científico sea posible. Al menos, esta es la lección que podemos extraer del cambio teórico que la física teórica sufrió durante el siglo XIX al transitar de un marco espacio-temporal newtoniano-galileano a otro marco dirigido por la geometría no-euclidiana que tuvo éxito al ser aplicada a la nueva teoría de la relatividad desarrollada por Einstein (Friedman, 1996, p. 389).

Por último, como señalamos en el argumento b.1 de la estrategia anterior, las pasadas generaciones de científicos seguramente consideraban que las técnicas y controles metodológicos que utilizaron eran más "estrictas" —y en este sentido mejores - que las técnicas y controles metodológicos utilizados por las generaciones que les precedieron. Sin embargo, como señalamos, cada generación puede construir un argumento similar sin socavar IP. Y dado que este patrón argumentativo puede repetirse ad nauseam, los realistas se han visto orillados a elaborar una estrategia diferente para socavar IP, a saber, mostrar que IP es un argumento falaz en sí mismo. En la siguiente y última sección de este texto analizaremos esta tercera estrategia realista.

\section{c. Mostrar que IP es un argumento falaz}

Argumento realista c. 1: ante la falta de éxito de las dos anteriores estrategias realistas para socavar IP, se ha tratado de mostrar que IP es un argumento falaz en sí mismo. Este argumento lo han suscrito autores como Lewis para mostrar que IP es un argumento falaz, Lewis sostiene que:

a. La mayoría de las teorías científicas actuales son verdaderas.

b. La mayoría de las teorías científicas pasadas difieren de las teorías actuales de manera significativa.

Por lo tanto, por inducción:

c. La mayoría de las teorías pasadas son falsas (Lewis, 2001, p. 372).

Si la pregunta es por qué las teorías científicas del pasado son falsas, la conclusión c), argumentan los realistas, claramente comete una petición de principio, a saber, las teorías del pasado son falsas porque las teorías actuales son verdaderas. 
Contrargumento IP al argumento realista c. 1: para bloquear este argumento, lo primero que debemos notar es que su estrategia consiste en invertir el procedimiento inferencial desarrollado por IP. Lewis pasa de la consideración hipotética de que las teorías científicas actuales son verdaderas, a la afirmación de que, si este es el caso, entonces las teorías del pasado deben ser falsas. Sin embargo, ninguno de los autores que defienden IP que hemos revisado hasta aquí ha sostenido que las teorías actuales son verdaderas. Por el contrario, se presume que muy probablemente las teorías científicas actuales no sean ni siquiera aproximadamente verdaderas ${ }^{9}$.

Por otro lado, diferir de manera "significativa" no necesariamente implica que las teorías científicas pasadas y las actuales exhiban valores de verdad distintos. Por ejemplo, una teoría científica puede ser abandonada o desechada por exhibir menos contenido de verdad que otra y no necesariamente por ser falsa. En este sentido, bien puede ser el caso que una teoría científica $T_{2}$ sea considerada como más aproximada a la verdad que la teoría $T_{1} y$, sobre este fundamento, decidir abandonar $\mathrm{T}_{1}{ }^{10}$.

Ahora bien, si concedemos que diferir de manera significativa debe ser entendido como "diferir en sus valores de verdad", el argumento de Lewis puede ser reformulado de la siguiente manera: argumento realista c. 2 .

a. La mayoría de las teorías científicas actuales son verdaderas.

b. La mayoría de las teorías científicas pasadas difieren de las teorías actuales de manera significativa.

c. Diferir de manera significativa quiere decir diferir en sus valores de verdad.

Por lo tanto, por inducción:

d. La mayoría de las teorías pasadas son falsas (Lewis, 2001, p. 372).

Una vez concedido el argumento c.2 los realistas pueden construir un argumento definitivo: argumento realista c.3.

e) Los científicos abandonan una teoría $T_{1}$ en favor de otra teoría $T_{2}$ si y solo si ellos creen que ambas difieren de manera significativa.

f) Diferir de manera significativa quiere decir diferir en sus valores de verdad.

g) $T_{1}$ ha sido abandonada por los científicos a favor de $T_{2}$.

h) $\mathrm{T}_{2}$ es considerada verdadera.

Por lo tanto, por inducción:

i) $T_{1}$ es considerada falsa.

\footnotetext{
${ }^{9}$ Véase por ejemplo Devitt, 2007 y Frost-Arnold, 2011.

${ }^{10}$ Para revisar algunas posturas sobre el incremento de la verosimilitud de las teorías científicas, véase Niiniluoto (2014) y Cevolani y Tambolo (2012).
} 
Contrargumento IP a los argumentos realistas c. 2 y c.3: para bloquear ambos argumentos, debemos recordar que las razones para abandonar una teoría específica ciertamente pueden ser diversas. Por ejemplo, una teoría científica puede ser abandonada por no ser suficientemente comprehensiva, por no estar suficientemente confirmada, por no ejercer la suficiente influencia en la comunidad científica o por ser incompleta. En otras palabras, el abandono de una teoría científica no puede reducirse causalmente a no ser verdadera. Basta recordar a este respecto la teoría del flogisto y la teoría calórica que citamos anteriormente. Como vimos, ambas teorías exhibieron ciertos éxitos empíricos a pesar de que sus términos teóricos constitutivos no tuvieron referencia alguna.

Argumento realista c.4: sin embargo, los realistas como Lewis todavía pueden argumentar que teorías como la del flogisto o la teoría calórica, en efecto pudieron exhibir cierto éxito empírico a pesar de ser teorías cuyos términos teóricos centrales no tuvieron referencia alguna. Sin embargo, los éxitos empíricos de este tipo de teorías en realidad son irrelevantes dado que ambas teorías fueron abandonadas. Y dado que ambas teorías no contenían términos con referencias genuinas, IP debe considerarlas falsas, cometiendo una petición de principio.

Contrargumento IP al argumento realista c.4: para bloquear este último argumento realista, los defensores de IP pueden argumentar que un término teórico que no tiene referencia en realidad no expresa ningún contenido semántico, esto es, no es ni verdadero ni falso. Si este anti-realismo semántico es el caso, las proposiciones atómicas que contienen nombres o clases naturales de términos que no refieren simplemente carecen de valores de verdad. Por supuesto, la lista de razones por las que un término teórico no exhibe referencia alguna en un momento específico, es larga. Mencionaré solo tres ejemplos:

-Por ignorancia en $t_{1}$

-Por una deficiente información en $\mathrm{t}_{1}$

-Por imposibilidad tecnológica en $\mathrm{t}_{1}$, etc.

Sin embargo, nada nos impide pensar desde un punto de vista lógico, que en un futuro tales términos obtengan su referencia genuina, esto es, que las proposiciones científicas que actualmente poseen términos teóricos parciales o vagos, se completen exitosamente a partir de la construcción de alternativas teóricas futuras. Si esto es posible, carecer de valores de verdad no convierte a una teoría científica en una teoría falsa. 
Sostengo que los defensores de IP pueden aceptar este tipo de anti-realismo semántico sin detrimento de su postura porque su tesis central es de carácter retrospectivo y no de carácter hipotético ni contrafáctico, como sí lo es el argumento construido por Lewis. La esencia del argumento IP no se expresa sobre cómo pudo ser la ciencia; sino cómo de hecho ha sido desde una interpretación estrictamente histórica y cómo puede llegar a ser desde una perspectiva estrictamente lógica.

\section{Conclusiones}

Para abordar el debate entre algunas versiones de RC y los defensores de IP, en este texto analizamos tres estrategias realistas que pretenden socavar IP. La primera estrategia trata de minimizar la relevancia epistémica que tiene el hecho de que la mayoría de las teorías exitosas del pasado que exhibieron cierto éxito empírico, hoy son consideradas estrictamente falsas. La segunda estrategia consiste en distinguir los llamados working posits o partes esenciales de las teorías que se han conservado durante el cambio teórico. Sin embargo, dado que mostramos que ninguna de estas dos estrategias logró contrarrestar IP, los realistas trataron de mostrar que IP es un argumento falaz en sí mismo al afirmar que si las teorías actuales son verdaderas, entonces las teorías del pasado deben ser falsas. No obstante, a este respecto mostramos que ninguna de las versiones de IP que revisamos implica considerar a las teorías actuales como verdaderas. Asimismo, tampoco pretenden establecer que la única razón para abandonar una teoría es que esta sea falsa. Como señalamos, las causas para abandonar una teoría son plurales e, incluso, pueden ser razones de naturaleza no epistémica.

No obstante, un punto débil de IP es que a pesar de que esta postura puede adoptar un anti-realismo semántico de la referencia y permitir que las teorías científicas actualmente parciales o deficientemente formuladas sean completadas exitosamente en un futuro; la interpretación retrospectiva y la inferencia prospectiva esenciales al argumento de la inducción pesimista no nos proporcionan las herramientas lógicas suficientes para calcular la probabilidad de éxito de tales teorías. Calcular esta probabilidad es, sin duda, una línea abierta para investigaciones futuras sobre el tema.

\section{Referencias}

Bird, A. (2007). What Is Scientific Progress? Nous, 41, 92-117.

Cevolani, G. y Tambolo, L. (2012). Progress as Approximation to the Truth: A Defence of the Verisimilitudinarian Approach. Erkenntnis, 78, 921-935.

Chang, H. (2003). Preservative Realism and Its Discontents: Revisiting Caloric. Philosophy of Science, 70(5), 902-912. 
De Solla, D. (1963). Little science, big science... and beyond. New York: Columbia University Press.

Devitt, M. (2007). Scientific Realism. En F. Jackson y M. Smith (eds.), The Oxford Handbook of Contemporary Philosophy. Oxford: Oxford University Press.

Fahrbach, L. (2011). How the growth of science ends theory change. Synthese, 180, 139-155.

Friedman, M. (1996). Objectivity and History. Review work of The Advancement of Science: Science without Legend, Objectivity without Illusions by Philip Kitcher. Erkenntnis, 44(3), 379-395.

Frost-Arnold, G. (2011). From the Pessimistic Induction to Semantic Antirealism. Philosophy of Science, 78(5), 1131-1142.

Held, C. (2011). Truth does not explain predictive success. Analysis, 41(2), 232234.

Kitcher, P. (1995). Précis of The Advancement of Science. Philosophy and Phenomenological Research, 55(3), 611-617.

Laudan, L. (1977). Progress and Its Problems, Towards a Theory of Scientific Growth. Berkeley: University of California Press.

Laudan, L. (1981). A Confutation of Convergent Realism. Philosophy of Science, 48(1), 19-49.

Leplin, J. (1992). Realism and Methodological Change. Proceedings of the Biennial Meeting of the Philosophy of Science Association, 1992(1), 435-445.

Lewis, P. (2001). Why the Pessimistic Induction is a Fallacy. Synthese, 129, 371380.

McMullin, E. (1984). A Case for Scientific Realism. En J. Leplin (ed.), Scientific Realism. Berkeley: University of California Press.

Mizrahi, M. (2013). The Pessimistic Induction: A Bad Argument Gone too Far. Synthese, 190, 3209-3226.

Niiniluoto, I. (2014). Scientific Progress as Increasing Verisimilitude. Studies in History and Philosophy of Science, 46, 73-77. 
Park, S. (2014). A Pessimistic Induction against Scientific Antirealism. Organon F, 21(1), 3-21.

Poincaré, H. (1905). Science and Hypothesis. New York: The Walter Scott Publishing Co.

Psillos, S. (1999). Scientific realism: how science tracks truth. London: Routledge.

Putnam, H. (1975). Mathematics Matter and Method. Philosophical Papers Volume I. Cambridge: Cambridge University Press.

Putnam, H. (1978). Meaning and the Moral Sciences. London: Routledge \& K. Paul.

Stanford, K. (2006). Exceeding our grasp: Science, history, and the problem of unconceived alternatives. Oxford: Oxford University Press.

Vickers, P. (2017). Understanding the Selective Realist Defence Against the PMI. Synthese, 194, 3221-3232.

Worrall, J. (1989). Structural realism: The best of both worlds. Dialectica, 43, 99124.

Wray, B. (2013). Pessimistic induction and the exponential growth of science reassessed. Synthese, 190, 4321-4330. 